\title{
Assensus
}

Revista Assensus Vol 2, Núm 3

Septiembre - Diciembre 2017

ISSN 2619-3884

Recepción: Noviembre 05 de 2016

Aceptación: Marzo 05 de 2017

Publicación: Septiembre 01 de 2017

\section{Incidencia de las habilidades sociales en el desempeño académico de estudiantes de sexto grado.}

Incidence of social skills in the academic performance of sixth grade students.

Gloria María Contreras

Universidad de Sucre - Colombia yoyicontreras2007@hotmail.com

Olga María Benítez

Universidad de Sucre - Colombia olgamarbenitez@hotmail.com

Tulio Amaya De Armas

Universidad de Sucre - Colombia tuamal@hotmail.com 


\section{Assensus}

\section{Resumen}

En este trabajo se evaluó la incidencia de las habilidades sociales: empatía, asertividad, trabajo en equipo y autoestima en el desempeño académico de estudiantes de sexto grado. La investigación se enmarca en un enfoque mixto (Creswell, 2009) puesto que en ella se combinan técnicas y métodos de investigación cuantitativos y cualitativos. La muestra la conformaron 155 estudiantes de sexto grado de dos instituciones del municipio de Sincelejo. Los resultados evidencian que el nivel de desarrollo de las habilidades sociales trabajo en equipo y asertividad son bastante heterogéneas tanto intra como inter grupos, es decir, su nivel de desarrollo no guarda una tendencia clara en ninguno de los dos grupos, mientras que autoestima y empatía tienen una tendencia entre en desarrollo a bien desarrolladas. Se encontró asociación estadísticamente significativa entre los desempeños logrados por los estudiantes en las áreas de matemáticas y de Ciencias Naturales, con la institución de donde provenían, lo que quiere decir que las calificaciones de los estudiantes de cada institución tendieron a agruparse alrededor de los mismos niveles de desempeño, pero diferenciables entre los grupo, ligeramente mejores para los de la institución privada. Además, el desempeño académico de los estudiantes en las áreas de Matemáticas y Ciencias Naturales, guarda cierta relación con el estado de desarrollo de las habilidades Autoestima y Empatía.

\section{Abstract}

In this work we evaluated the incidence of social skills: empathy, assertiveness, teamwork and self-esteem in the academic performance of students the sixth grade. The research is part of a mixed approach (Creswell, 2009) since it combines quantitative and qualitative research methods and techniques. 155 sixth grade students from two institutions of the municipality of Sincelejo formed the sample. The results show that the level of development of social work skills teamwork and assertiveness are by quite heterogeneous both intra as inter groups, i.e., their level of development is a clear trend in none of the two groups, while it self-esteem and empathy have a tendency from developed to well-developed. We found a statistically significant association between the performances achieved by the students in the areas of mathematics and natural sciences, with the institution where they came from, which means that the qualifications of students from each institution tended to gather around the same levels of performance, but differentiable among the group, slightly better for the private institution. In addition, the academic performance of students in the areas of mathematics and natural sciences, keep some relation with the State of development of self-esteem and empathy skills.

\section{Palabras Clave}

Habilidades sociales, desempeño académico, asertividad, empatía, autoestima.

\section{Keywords}

social skills, academic performance, assertiveness, empathy and self-esteem. 


\section{Assensus}

\section{Introducción}

El desarrollo de habilidades sociales, entendidas como "las capacidades o destrezas sociales específicas requeridas para ejecutar competentemente una tarea interpersonal" (Monjas, 2000, P.28), propicia procesos de interacción social los cuales son, por naturaleza, formadores de la integridad personal de los individuos que los vivencian. Interacciones que propician procesos formativos y funcionan como el conjunto de necesidades básicas, que al ser satisfechas tienen grandes repercusiones en la solución de problemas "como: el fracaso y abandono escolar, la delincuencia y otros tipos de problemas en la vida adulta" (García et al., 2013, p. 146), que bien podrían evitarse con la integración temprana de la persona a un grupo social, donde sea aceptado. Monjas (2000) se refiere a las habilidades, como un conjunto de conductas aprendidas, objeto de la educación social en la cual se pretende el perfeccionamiento del hombre en todas sus dimensiones. Lo anterior tiene asidero si se considera que la persona es un ser social, moldeable por la educación, y la educación es, por naturaleza, un medio óptimo de socialización que afecta el desarrollo cognoscitivo, el desarrollo de la conducta y el desarrollo afectivo de las personas inmersas en un proceso educativo (Damián, Muria, 2006). Y en que además, la sociedad de hoy exige personas asertivas, emprendedoras, líderes que sean capaces de tomar decisiones e implementar formas de generar recursos por cuenta propia.

En el mismo sentido, la globalización exige de las personas, cada vez mayores habilidades para relacionarse en su entorno, tanto de trabajo, familiar y social, lo que significa que las competencias específicas para el desarrollo de una profesión en el mercado laboral ya no son suficientes (Balseiro et al., 2012) y que el mundo exige de las personas sensibilidad ante la dificultad ajena, solidaridad con el necesitado, pero sobre todo confianza en sí mismo para realizar de la mejor manera, lo que sabe hacer trabajando en equipo con sus compañeros.

Las acciones del diario vivir colombiano, como la violencia, el conflicto, la falta de convivencia pacífica y la intolerancia son consecuencia de la crisis de valores que afronta la sociedad actual (Pascual, 2000), por lo que habría de esperarse que en la familia y en especial en las Instituciones Educativas existiera una creciente crisis de valores reflejada en las relaciones interpersonales, es decir, las habilidades sociales parecen estar en crisis en los estamentos primarios de la sociedad colombiana. En consecuencia, para mejorar tal situación, los Proyectos Educativos Institucionales (PEI), los proyectos transversales y la reglamentación que provee el Ministerio de Educación Nacional (MEN), recomiendan como vía de solución el desarrollo de habilidades sociales, pues constituyen el Saber Ser, que es según la Unesco (2008) uno de los pilares fundamentales de la educación, que le facilita a las personas aprender a convivir en sociedad.

Durante la última década, el sistema educativo colombiano se ha visto abocado a cambios fundamentales en cuanto a políticas de mejoramiento de la calidad educativa, encaminándose hacia un enfoque basado en competencias, donde se supone que el estudiante adquiere aprendizajes que le ayuden a resolver problemas de y para la vida (Trujillo, 2011). Tratando de enrumbarse por 
esta vía, las instituciones educativas acogiendo políticas estatales, elaboran estrategias para estimular entre estudiantes y demás miembros de la comunidad educativa, relaciones óptimas entre pares, donde prime la convivencia y la solidaridad. Sin embargo, estos esfuerzos serían infructuosos sino se llevan a cabo a través de acciones formativas, donde se les haga un seguimiento y evalúen periódicamente con el fin de mejorarlos cada vez más, de forma que las habilidades sociales se den con naturalidad entre los miembros de la comunidad educativa y contribuyan a una sana convivencia entre ellos. Algunos de los fracasos institucionales en este sentido, pueden deberse a que se deja de lado el desarrollo de ciertas habilidades que aunque no son evaluadas en este ámbito si son muy importantes en el desarrollo integral de las personas.

A propósito, Ortega (2005, p.125) sostiene que “la educación abarca toda la vida y ámbitos de la sociedad, la escuela no debe solo preocuparse por transmitir conocimientos, sino por la educación social que posibilita la convivencia abierta y global". En este sentido, Durán, Flores y Valdebenito (2015), recomiendan potencializar las habilidades sociales como herramientas que se pueden potenciar a través de talleres, que fomenten el trabajo en equipo y colaborativo, y actividades en que se incentive la comunicación entre los estudiantes. Estos autores creen que es posible hacerlo desde cualquier área y no sólo desde un área o espacio pedagógico específico como es el caso de las asignaturas de Ética o religión, que se consideran, de acuerdo a las creencias de algunos docentes, las únicas áreas en las que se deben potenciar este tipo de habilidades.

En los planteamientos de Durán, Flores y Valdebenito (2015) se vislumbra, que en la escuela se privilegie el aprendizaje colaborativo, el cual hace referencia al desarrollo de actividades de aula, en pequeños grupos. Es un trabajo centrado en los estudiantes, donde ellos forman pequeños equipos centrados en resolver una tarea que generalmente propone el docente. Los estudiantes trabajan colaborativamente en la tarea, interactuando e intercambiando información hasta que cada miembro del equipo llega a comprenderla. En este proceso los estudiantes son responsables de su propio aprendizaje. Según López (2009) para desarrollar con éxito este proceso es necesario que cada estudiante "pueda valorar sus progresos y reconocer sus dificultades y posibilidades, permitiendo así una mejor autoregulación del aprendizaje (...) el conocimiento es concebido como un constructo social, facilitado por la interacción, la evaluación y la cooperación entre iguales" (p.94).

Al respecto Godino (2013, p.124) considera, que "La interacción entre los estudiantes y entre los estudiantes y el profesor puede provocar que cada uno reflexione a partir de lo que aportan los demás y así poder alcanzar niveles más altos de comprensión". Esto es de tanta importancia en los procesos de aprendizaje, que con el aprendizaje colaborativo se promueven los dos pilares de la educación más difíciles de desarrollar en la escuela: aprender a aprender y aprender a vivir juntos (Unesco, 2008), debido quizás al mismo proceso interactivo, donde se fomentan tanto competencias como valores, lo que conllevaría a formar alumnos más humanos, pero a la vez, altamente competitivos. 
El problema que se ha detectado en los estudiantes en general es que no saben responder ante escenarios de incertidumbre, donde hay que romper paradigmas y adaptarse al cambio. Sólo se les ha entregado muchas habilidades y herramientas que solo dan respuestas a escenarios específicos, ya aprobados y conocidos. El sistema educativo colombiano está muy enfocado en el desarrollo de habilidades cognitivas, pero ¿qué sucede con el desarrollo de habilidades sociales? Los sistemas de evaluación externos y las pruebas de admisión a las universidades siguen siendo de selección múltiple que miden contenidos y conocimientos, dejando de lado la integralidad del ser, que concierne a la forma como los sujetos resuelven los problemas y cómo se relacionan con los demás. Cuando entran en el campo laboral se hace una entrevista, se hacen pruebas psicotécnicas que evalúan las habilidades sociales y de estas depende la permanencia en una universidad, la forma cómo se enfrentan las dificultades, cómo se comunican efectivamente en diversas situaciones y el emprendimiento que manifiesten, y por tanto la continuidad en un trabajo.

En este trabajo se tuvo como objetivo evaluar la incidencia de las habilidades sociales: empatía, asertividad, trabajo en equipo y autoestima en el desempeño académico de estudiantes del grado sexto de las instituciones Educativas San Vicente de Paul y el Centro Educativo Liceo Panamericano, de la ciudad de Sincelejo. El trabajo en equipo se refiere al trabajo realizado por varios individuos en busca de un objetivo común, en un ambiente de armonía, obteniéndose resultados beneficiosos para todos. La autoestima hace referencia a la percepción evaluativa que cada persona tiene sobre sí mismo, para Monjas y González (2000, p.25) "es la evaluación que hace el individuo de sí mismo y que tiende a mantenerse; expresa una actitud de aprobación o rechazo y hasta qué punto el sujeto se considera capaz, significativo, exitoso y valioso". En definitiva, la autoestima es la valoración, ya sea positiva o negativa, que una persona hace de sí mismo. La asertividad tiene que ver con el conocimiento que se tiene de los derechos y la opción de defenderlos, reconociendo y respetando los derechos de Ios demás. En palabra de Monjas et al., (1998) es la conducta interpersonal que implica directamente sentimientos de la persona y la defensa los derechos personales, reconociendo los derechos ajenos. Y empatía está relacionada con la intervención afectiva de una persona en una realidad que afecta a otras personas. Según Rey (2003, p.186) “La empatía es una habilidad social de alto nivel, que hace referencia a la capacidad de comprender los sentimientos de otra persona, o compartir la emoción percibida en el otro".

\section{Aspectos Metodológicos}

Esta investigación se enmarca en un enfoque mixto (Creswell, 2009) puesto que en ella se combinan técnicas y métodos de investigación cuantitativos y cualitativos. Se consideran variables cuantitativas como las notas de los estudiantes en las asignaturas de Matemáticas, Lengua Castellana y Ciencias Naturales, y cualitativas como las opiniones de los estudiantes al responder la encuesta sobre sus habilidades sociales. Respuestas que fueron cuantificadas, donde las categorías creadas van de 1 a 5 , donde el 1 corresponde a nunca y el 5 a siempre hacer la cuestión por la que se les indagó en ese ítem; por lo que el 


\section{Assensus}

tratamiento que se hace de estas variables es meramente cuantitativo. Para medir el estado de desarrollo de cada habilidad se tendrá en cuenta la siguiente convención: una habilidad es considerada insipiente cuando las respuestas están entre nunca y casi nunca, en desarrollo cuando están entre a veces y casi siempre y desarrollada cuando son siempre. Las respuestas dadas en la entrevista se conservan y se tratan cualitativamente. La muestra la conformaron 155 estudiantes de sexto grado: 79 de la institución Educativa San Vicente de Paul (de carácter oficial) y 76 del Centro Educativo Liceo Panamericano (de carácter privado).

Se utilizaron las notas correspondientes al primer semestre, en las asignaturas de Matemáticas, Lengua Castellana y Ciencias naturales, de los estudiantes de las dos instituciones incluidas en la muestra y además, se aplicó un cuestionario, el cual fue validado previamente (Monjas et al., 1998; Monjas y González 2000; Rey, 2003), y está compuesto por 52 ítems, relacionados con las cuatro habilidades sociales siguientes: trabajo en equipo 11 ítems, autoestima 10 ítems, asertividad 17 ítems y empatía 14 ítems.

Para analizar la fiabilidad del cuestionario se utilizó el coeficiente Alfa de Cronbach. El coeficiente Alfa de Cronbach para los constructos que tienen que ver con trabajo en equipo fue de 0.805 , para los autoestima fue de 0.86 , para los de asertividad de 0,801, para los de empatía fue de 0,827 y para los del cuestionario completo de 0.836, como puede apreciarse en la tabla 1.

Tabla 1. Estadísticos de fiabilidad para el cuestionario.

\begin{tabular}{lcc} 
Constructo & $\begin{array}{c}\text { Alfa de } \\
\text { Cronbach }\end{array}$ & $\begin{array}{c}\text { N de } \\
\text { elementos }\end{array}$ \\
\hline Trabajo en equipo & 0.805 & 11 \\
Autoestima & 0.805 & 10 \\
Asertividad & 0,801 & 17 \\
Empatía & 0,827 & 14 \\
\hline Total & 0.836 & 52
\end{tabular}

Fuente: Contreras, Benítez \& Amaya De Armas (2016).

Lo que quiere decir que el nivel de correlación de las variables en el instrumento es adecuado con el instrumento como un todo, lo que denota a priori una buena consistencia interna de dicho instrumento (García, González y Ballesteros, 2001; Hernández, Fernández y Baptista, 2010). Para evaluar la validez del constructo el comportamiento psicométrico de las características trabajo en equipo, autoestima, asertividad y empatía se utilizó el coeficiente $\mathrm{KMO}$. El valor encontrado para el KMO fue adecuado ( $\mathrm{KMO}=0.795, \mathrm{P}<0.05)$, con todas las comunalidades mayores que 0.4 (Gil-Pascual, 2009), lo que significa que las variables en su conjunto representan adecuadamente el concepto estudiado.

Los procedimientos estadísticos realizados fueron: 1) el cálculo de las tablas de contingencia asociadas y el coeficiente Chi cuadrado de Pearson $\left(\chi^{2}\right)$ correspondiente, usados para medir la significatividad de las asociaciones de las notas de Matemáticas, Castellano y Ciencias naturales con los constructos trabajo en equipo, autoestima, asertividad y empatía entre los grupos, 2) el cálculo del 


\section{Assensus}

coeficiente Alfa de Cronbach para analizar la fiabilidad del instrumento y 3) un análisis factorial para determinar la validez de constructo y el comportamiento psicométrico de las características trabajo en equipo, autoestima, asertividad y empatía en el instrumento.

\section{Resultados y discusión}

\section{Desempeño por institución}

En la tabla 2 se puede apreciar que los desempeños de los estudiantes en el área de Matemáticas se agrupan alrededor de bajo $(37,97 \%)$ y básico $(48,10 \%)$ para los de San Vicente de Paul y alrededor de básico $(59,21 \%)$, y alto $(30,26 \%)$ para los del Centro Educativo Liceo Panamericano. Además, se encontró asociación estadísticamente significativa $(\chi 2=22,776, p<0,05)$ entre el desempeño logrado en el área de matemáticas, con la institución de donde provenían los estudiantes, es decir, las calificaciones de los estudiantes de cada institución tendieron a agruparse alrededor de los mismos niveles de desempeño: entre bajo y básico para San Vicente y entre Básico y alto para el Centro Educativo Liceo Panamericano.

Tabla 2. Desempeño académico en Matemáticas por institución.

\begin{tabular}{llrrrrr}
\hline & \multicolumn{3}{c}{ Notas de matemáticas } & \multicolumn{1}{c}{ Total } \\
\cline { 3 - 6 } & \multicolumn{2}{c}{ Bajo } & Básico & Alto & Superior & \multicolumn{1}{c}{ Totar } \\
\hline Institución & San Vicente & 30 & 38 & 11 & 0 & 79 \\
& Centro Educativo Liceo & 7 & 45 & 23 & 1 & 76 \\
Total & Panamericano & 37 & 83 & 34 & 1 & 155 \\
Asociación & $\chi^{2}=22,776$ & & $\mathrm{P}<0,05$ & Sig. 0.000 & \\
\hline
\end{tabular}

Fuente: Contreras, Benítez \& Amaya De Armas (2016).

En la tabla 3 se aprecia que los desempeños de los estudiantes en el área de Lengua Castellana son bastante heterogéneos tanto para los de San Vicente de Paul como para los del Centro Educativo Liceo Panamericano. No se encontraron evidencias estadísticas de asociación $\left(\chi^{2}=3,820, p>0,05\right)$ entre el desempeño logrado en el área de castellano con la institución de donde provenían los estudiantes, es decir, los desempeños de los estudiantes de cada institución no mostraron una tendencia hacia algún nivel específico de desempeño.

Tabla 3. Desempeño académico en Lengua Castellana por institución.

\begin{tabular}{|c|c|c|c|c|c|c|}
\hline & & \multicolumn{4}{|c|}{ Notas de matemáticas } & \multirow[b]{2}{*}{ Total } \\
\hline & & Bajo & Básico & Alto & Superior & \\
\hline \multirow[t]{2}{*}{ Institución } & \multirow{4}{*}{$\begin{array}{l}\text { San Vicente } \\
\text { Centro Educativo Liceo } \\
\text { Panamericano }\end{array}$} & 23 & 47 & 9 & 0 & 79 \\
\hline & & 19 & 41 & 14 & 2 & 76 \\
\hline \multirow{2}{*}{$\begin{array}{l}\text { Total } \\
\text { Asociación }\end{array}$} & & 37 & 42 & 88 & 23 & 155 \\
\hline & & $\chi^{2}=5,540$ & & 0,05 & Sig. 0.282 & \\
\hline
\end{tabular}




\section{Assensus}

Como se puede apreciar en la tabla 4, los desempeños de los estudiantes en el área de Ciencias Naturales están alrededor de básico $(65,82 \%$ y 42,10\% respectivamente) y alto (18,98\% y $57,89 \%$ respectivamente) en los estudiantes de ambas instituciones; observándose, leves mejores niveles de desempeños en los estudiantes del Centro Educativo Liceo Panamericano. Además, se encontró asociación estadísticamente significativa $(\chi 2=30,970, p<0,05)$ entre el desempeño logrado en esta área, con la institución de proveniencia de los estudiantes.

Tabla 4. Desempeño académico en Ciencias Naturales por institución.

\begin{tabular}{|c|c|c|c|c|c|c|}
\hline & \multicolumn{4}{|c|}{ Notas de matemáticas } & \multirow[b]{2}{*}{ Total } \\
\hline & & Bajo & Básico & Alto & Superior & \\
\hline \multirow[t]{2}{*}{ Institución } & San Vicente & 12 & 52 & 15 & 0 & 79 \\
\hline & $\begin{array}{l}\text { Centro Educativo Liceo } \\
\text { Panamericano }\end{array}$ & 0 & 32 & 44 & 0 & 76 \\
\hline \multirow{2}{*}{$\begin{array}{l}\text { Total } \\
\text { Asociación }\end{array}$} & & 12 & 84 & 59 & 0 & 155 \\
\hline & & $\chi^{2}=30,970$ & & $<0,05$ & Sig. 0.000 & \\
\hline
\end{tabular}

Fuente: Contreras, Benítez \& Amaya De Armas (2016).

Las habilidades sociales en los estudiantes de ambos grupos son muy heterogéneas en la mayoría de los constructos analizados: en Trabajo en equipo, sólo el constructo "acordar dividirse el trabajo de forma proporcional" resultó con asociaciones significativas $(\chi 2=10,676, P<0,05)$ y bien desarrollado en ambos grupos. En Autoestima sólo en los constructos "le gustaría poder sentir más respeto por sí mismo" y "en general, inclinarse a pensar que se es un fracasado" se encontraron asociaciones estadísticamente significativas $(\chi 2=24,089$ y $\chi 2=$ $15,901$ respectivamente, con $\mathrm{P}<0,05)$, con las habilidades sociales en desarrollo. En Asertividad, sólo en los constructos "disculparse cuando se comete un error", "hacer preguntas de tipo personal a los demás", "ser capaz de conversar con personas desconocidas", "poder ser amigo de alguien que te ha rechazado anteriormente" y "pedir que te devuelvan las cosas que has prestado" se encontraron altos niveles de homogeneidad entre las respuestas intra grupos $(\chi 2$ $=11,605 ; \chi_{2}=13,243 ; \chi 2=9,399 ; \chi_{2}=15,607 ; \chi_{2}=9,585$, respectivamente, con $\mathrm{P}<0,05)$, y además, el estado manifestados de las habilidades fue incipiente en estos constructos, en ambos grupos y muy heterogéneas en los demás. Mientras que en Empatía sólo en los constructos "cuando lo necesitan, animo a mis amigos y compañeros" y "hablo muy bien, tengo facilidad para hablar" se encontraron altos niveles de homogeneidad en las respuestas intra grupos $(\chi 2$ $=9,934 ; \chi 2=13,130$, respectivamente, con $\mathrm{P}<0,05)$, y para el caso, también inter grupos, y además, el estado manifiesto de las habilidades está en desarrollo en los grupos de ambas instituciones.

\section{Trabajo en Equipo.}

En esta habilidad constructos como "estar de acuerdo en que antes de entregar la tarea, todos los miembros del equipo revisen el trabajo" y "cumplir con las tareas y compromisos que quedan a su cargo", presentaron altos niveles de homogeneidad en las respuestas asociadas a los niveles de desempeños de quienes las dieron, en cada una de las tres áreas analizadas (ver $\chi 2$ y p-value 


\section{Assensus}

$<0,05$ en la tabla 6). Es decir, las respuestas de los estudiantes, en cada área, guardan una estrecha relación con el grado de acuerdo sobre realizar o no este tipo de acciones para resolver las tareas. Además, estos dos constructos de la habilidad trabajo en equipo se encuentran en desarrollo en el $41,3 \%$ de los estudiantes y bien desarrollados en el $53,5 \%$ de ellos, y los desempeños en las tres áreas se agrupan alrededor del nivel básico, como puede apreciarse en la tabla 5.

Tabla 5. Resumen de niveles de desempeño en las tres áreas contra grado de desarrollo del constructo cumplir con las tareas y compromisos que quedan a su cargo.

\begin{tabular}{|c|c|c|c|c|c|c|c|}
\hline & & \multicolumn{5}{|c|}{$\begin{array}{l}\text { Cumplir con las tareas y compromisos que quedan a } \\
\text { su cargo }\end{array}$} & \multirow[b]{2}{*}{ Total } \\
\hline & & Nunca & Casi Nunca & $\begin{array}{c}\text { A } \\
\text { veces }\end{array}$ & $\begin{array}{l}\text { Casi } \\
\text { siempre }\end{array}$ & Siempre & \\
\hline \multirow{5}{*}{$\begin{array}{l}\text { Notas de } \\
\text { Matemáticas }\end{array}$} & Bajo & 0 & 1 & 18 & 6 & 12 & 37 \\
\hline & Básico & 3 & 2 & 10 & 26 & 42 & 83 \\
\hline & Alto & 1 & 1 & 2 & 2 & 28 & 34 \\
\hline & Superior & 0 & 0 & 0 & 0 & 1 & 1 \\
\hline & Total & 4 & 4 & 30 & 34 & 83 & 155 \\
\hline \multirow{4}{*}{$\begin{array}{l}\text { Notas de } \\
\text { ciencias } \\
\text { naturales }\end{array}$} & Bajo & 0 & 0 & 8 & 2 & 2 & 12 \\
\hline & Básico & 3 & 3 & 19 & 21 & 38 & 84 \\
\hline & Alto & 1 & 1 & 3 & 11 & 43 & 59 \\
\hline & Total & 4 & 4 & 30 & 34 & 83 & 155 \\
\hline \multirow{5}{*}{$\begin{array}{l}\text { Notas de } \\
\text { Castellano }\end{array}$} & Bajo & 1 & 1 & 13 & 14 & 13 & 42 \\
\hline & Básico & 3 & 3 & 15 & 16 & 51 & 88 \\
\hline & Alto & 0 & 0 & 2 & 4 & 17 & 23 \\
\hline & Superior & 0 & 0 & 0 & 0 & 2 & 2 \\
\hline & Total & 4 & 4 & 30 & 34 & 83 & 155 \\
\hline
\end{tabular}

Fuente: Contreras, Benítez \& Amaya De Armas (2016).

Constructos como "participar en discusiones acerca del mejor camino para llevar a cabo una tarea", "durante el desarrollo del trabajo en equipo, evito interrumpir y entorpecer el trabajo de mis compañeros" y "proponer soluciones para resolver los conflictos que puedan surgir en el equipo" presentaron altos niveles de homogeneidad en las respuestas asociadas a los niveles de desempeños de quienes las dieron, en cada una de dos de las tres áreas analizadas, como se puede apreciar en la tabla 6 . Lo que puede interpretarse como que la asociación entre las respuestas al interior de los grupos, se dieron con los niveles de desempeño de sólo dos de las asignaturas en estos tres constructos.

Mientras que los constructos "escuchar a los demás cuando participan", "acordar dividirse el trabajo de forma proporcional" y "asistir puntual a las reuniones para desarrollar el trabajo" presentaron altos niveles de homogeneidad en las respuestas asociadas a los niveles de desempeños de sólo una de las tres áreas analizadas, como puede apreciarse en la tabla 6. En términos generales, los niveles de desarrollo de la habilidad social trabajo en equipo es bastante heterogénea en los estudiantes de las dos instituciones de la muestra, siendo deseable, según Monjas (2014) que se aprenda y se llegue a trabajar como miembro de un equipo, que se llegue a ser fiel a un grupo y a sentirse parte de él, 


\section{Assensus} como una fortaleza para favorecer las relaciones interpersonales y generar una
mejora y un incremento de la calidad de los procesos que se generen.

Tabla 6. Chi cuadrado $\left(\chi^{2}\right)$ para habilidades sociales por asignaturas e institución de proveniencia.

\begin{tabular}{|c|c|c|c|c|}
\hline $\begin{array}{l}\text { Habilidad } \\
\text { social }\end{array}$ & Constructo & Mat. & Cast. & C. Nat. \\
\hline \multirow{11}{*}{$\begin{array}{l}\text { Trabajo en } \\
\text { equipo }\end{array}$} & $\begin{array}{l}\text { Participar en la discusión acerca del mejor } \\
\text { camino para llevarla a cabo una tarea }\end{array}$ & $\begin{array}{l}\chi^{2}=11,23 \\
P>0,05\end{array}$ & $\begin{array}{l}\chi^{2}=19,723 \\
P<0,05\end{array}$ & $\begin{array}{l}\chi^{2}=23,736 \\
P<0,05\end{array}$ \\
\hline & Escuchar a los demás cuando participan & $\begin{array}{l}\chi^{2}=25,858 \\
P<0,05\end{array}$ & $\begin{array}{l}\chi^{2}=7,503 \\
P>0,05\end{array}$ & $\begin{array}{l}\chi^{2}=7,346 \\
P>0,05\end{array}$ \\
\hline & Oponerse a las ideas de las demás & $\begin{array}{l}\chi^{2}=8,532 \\
P>0,05\end{array}$ & $\begin{array}{l}\chi^{2}=11,249 \\
P>0,05\end{array}$ & $\begin{array}{l}\chi^{2}=3,283 \\
P>0,05\end{array}$ \\
\hline & $\begin{array}{l}\text { Propiciar un ambiente agradable de } \\
\text { tolerancia y buen trato }\end{array}$ & $\begin{array}{l}\chi^{2}=7,865 \\
P>0,05\end{array}$ & $\begin{array}{l}\chi^{2}=8,992 \\
P>0,05\end{array}$ & $\begin{array}{l}\chi^{2}=8,398 \\
P>0,05\end{array}$ \\
\hline & $\begin{array}{l}\text { Acordar dividirse el trabajo de forma } \\
\text { proporcional }\end{array}$ & $\begin{array}{l}\chi^{2}=8,933 \\
P>0,05\end{array}$ & $\begin{array}{l}\chi^{2}=25,384 \\
P<0,05\end{array}$ & $\begin{array}{l}\chi^{2}=8,563 \\
P>0,05\end{array}$ \\
\hline & $\begin{array}{l}\text { Estar de acuerdo que antes de entregar la } \\
\text { tarea, todos los miembros del equipo } \\
\text { revisen el trabajo }\end{array}$ & $\begin{array}{l}\chi^{2}=26,638 \\
P<0,05\end{array}$ & $\begin{array}{l}\chi^{2}=24,189 \\
P<0,05\end{array}$ & $\begin{array}{l}\chi^{2}=28,488 \\
P<0,05\end{array}$ \\
\hline & $\begin{array}{l}\text { Haces sugerencias y recomendaciones } \\
\text { para hacer el trabajo }\end{array}$ & $\begin{array}{l}\chi^{2}=20,689 \\
P<0,05\end{array}$ & $\begin{array}{l}\chi^{2}=8,011 \\
P>0,05\end{array}$ & $\begin{array}{l}\chi^{2}=9,137 \\
P>0,05\end{array}$ \\
\hline & $\begin{array}{l}\text { Asistir puntual a las reuniones para } \\
\text { desarrollar el trabajo }\end{array}$ & $\begin{array}{l}\chi^{2}=12,364 \\
P>0,05\end{array}$ & $\begin{array}{l}\chi^{2}=28,698 \\
P<0,05\end{array}$ & $\begin{array}{l}\chi^{2}=13,093 \\
P>0,05\end{array}$ \\
\hline & $\begin{array}{l}\text { Durante el desarrollo del trabajo en } \\
\text { equipo, evito interrumpir y entorpecer el } \\
\text { trabajo de mis compañeros }\end{array}$ & $\begin{array}{l}\chi^{2}=11,249 \\
P>0,05\end{array}$ & $\begin{array}{l}\chi^{2}=27,363 \\
P<0,05\end{array}$ & $\begin{array}{l}\chi^{2}=25,911 \\
P<0,05\end{array}$ \\
\hline & $\begin{array}{l}\text { Cumplir con las tareas y compromisos que } \\
\text { quedan a su cargo }\end{array}$ & $\begin{array}{l}\chi^{2}=40,460 \\
P<0,05\end{array}$ & $\begin{array}{l}\chi^{2}=27,289 \\
P<0,05\end{array}$ & $\begin{array}{l}\chi^{2}=31,218 \\
P<0,05\end{array}$ \\
\hline & $\begin{array}{l}\text { Proponer soluciones para resolver los } \\
\text { conflictos que puedan surgir en el equipo }\end{array}$ & $\begin{array}{l}\chi^{2}=24,52 \\
P<0,05\end{array}$ & $\begin{array}{l}\chi^{2}=24,133 \\
P<0,05\end{array}$ & $\begin{array}{l}\chi^{2}=5,182 \\
P>0,05\end{array}$ \\
\hline \multirow{12}{*}{ Autoestima } & Estar satisfecho consigo mismo & $\begin{array}{l}\chi^{2}=7,650 \\
P>0,05\end{array}$ & $\begin{array}{l}\chi^{2}=6,792 \\
P>0,05\end{array}$ & $\begin{array}{l}\chi^{2}=6,616 \\
P>0,05\end{array}$ \\
\hline & Pensar que no se es buena persona & $\begin{array}{l}\chi^{2}=11,489 \\
P>0,05\end{array}$ & $\begin{array}{l}\chi^{2}=10,919 \\
P>0,05\end{array}$ & $\begin{array}{l}\chi^{2}=10,818 \\
P>0,05\end{array}$ \\
\hline & $\begin{array}{l}\text { Estar convencido de tener algunas buenas } \\
\text { cualidades }\end{array}$ & $\begin{array}{l}\chi^{2}=27,377 \\
P<0,05\end{array}$ & $\begin{array}{l}\chi^{2}=12,310 \\
P>0,05\end{array}$ & $\begin{array}{l}\chi^{2}=35,899 \\
P<0,05\end{array}$ \\
\hline & $\begin{array}{l}\text { Ser capaz de hacer las cosas tan bien } \\
\text { como la mayoría de la gente }\end{array}$ & $\begin{array}{l}\chi^{2}=25,516 \\
P<0,05\end{array}$ & $\begin{array}{l}\chi^{2}=27,456 \\
P<0,05\end{array}$ & $\begin{array}{l}\chi^{2}=35,745 \\
P<0,05\end{array}$ \\
\hline & $\begin{array}{l}\text { Sentir que no se tiene demasiadas cosas } \\
\text { de las que estar orgulloso }\end{array}$ & $\begin{array}{l}\chi^{2}=24,479 \\
P<0,05\end{array}$ & $\begin{array}{l}\chi^{2}=6,089 \\
P>0,05\end{array}$ & $\begin{array}{l}\chi^{2}=8,309 \\
P>0,05\end{array}$ \\
\hline & Sentirse realmente inútil & $\begin{array}{l}\chi^{2}=7,663 \\
P>0,05\end{array}$ & $\begin{array}{l}\chi^{2}=13,350 \\
P>0,05\end{array}$ & $\begin{array}{l}\chi^{2}=7,255 \\
P>0,05\end{array}$ \\
\hline & $\begin{array}{l}\text { Sentir que se es una persona digna de } \\
\text { aprecio, al menos en igual medida que los } \\
\text { demás }\end{array}$ & $\begin{array}{l}\chi^{2}=14,813 \\
P>0,05\end{array}$ & $\begin{array}{l}\chi^{2}=13,246 \\
P>0,05\end{array}$ & $\begin{array}{l}\chi^{2}=7,249 \\
P>0,05\end{array}$ \\
\hline & $\begin{array}{l}\text { Le gustaría poder sentir más respeto por } \\
\text { mí mismo }\end{array}$ & $\begin{array}{l}\chi^{2}=7,329 \\
P>0,05\end{array}$ & $\begin{array}{l}\chi^{2}=10,845 \\
P>0,05\end{array}$ & $\begin{array}{l}\chi^{2}=6,221 \\
P>0,05\end{array}$ \\
\hline & $\begin{array}{l}\text { En general, inclinarse a pensar que se es } \\
\text { un fracasado }\end{array}$ & $\begin{array}{l}\chi^{2}=7,836 \\
P>0,05\end{array}$ & $\begin{array}{l}\chi^{2}=5,444 \\
P>0,05\end{array}$ & $\begin{array}{l}\chi^{2}=5,931 \\
P>0,05\end{array}$ \\
\hline & Tener una actitud positiva hacia sí mismo & $\begin{array}{l}\chi^{2}=3,972 \\
P>0,05\end{array}$ & $\begin{array}{l}\chi^{2}=12,356 \\
P>0,05\end{array}$ & $\begin{array}{l}\chi^{2}=4,797 \\
P>0,05\end{array}$ \\
\hline & $\begin{array}{l}\text { Decir que no cuando le piden algo } \\
\text { prestado } \\
\text { Hacer elogios a los amigos }\end{array}$ & $\begin{array}{l}\chi^{2}=26,735 \\
P<0,05 \\
\chi^{2}=8,092 \\
P>0,05\end{array}$ & $\begin{array}{l}\chi^{2}=1,937 \\
P>0,05 \\
\chi^{2}=10,509 \\
P>0,05\end{array}$ & $\begin{array}{l}\chi^{2}=4,879 \\
P>0,05 \\
\chi^{2}=8,855 \\
P>0,05\end{array}$ \\
\hline & Cuando se necesita algo se pide el favor & $\begin{array}{l}\chi^{2}=26,101 \\
P<0,05\end{array}$ & $\begin{array}{l}\chi^{2}=25,608 \\
P<0,05\end{array}$ & $\begin{array}{l}\chi^{2}=11,359 \\
P>0,05\end{array}$ \\
\hline
\end{tabular}




\section{Assensus}

\begin{tabular}{|c|c|c|c|c|}
\hline Asertividad & $\begin{array}{l}\text { Disculparse cuando se comete un error } \\
\text { Aceptar cuando está asustado y se pide } \\
\text { ayuda } \\
\text { Si algo te molesta, lo haces saber } \\
\text { Aceptar que hay muchas cosas que no } \\
\text { sabes } \\
\text { Hacer preguntas de tipo personal a los } \\
\text { demás } \\
\text { Hacer críticas constructivas a los demás } \\
\text { Ser capaz de conversar con personas } \\
\text { desconocidas } \\
\text { Poder ser amigo de alguien que te ha } \\
\text { rechazado anteriormente } \\
\text { Cuando tienes dudas, pides que te las } \\
\text { aclaren } \\
\text { Cuando alguien te cae bien, se lo dices } \\
\text { Ser capaz de exigir ser atendido cuando te } \\
\text { han hecho esperar mucho tiempo } \\
\text { Ser capaz de expresar su opinión aunque } \\
\text { sea diferente a la de los demás } \\
\text { Pedir que te devuelvan las cosas que has } \\
\text { prestado } \\
\text { Si alguien te está molestando en público, } \\
\text { le pedirle que deje de hacerlo }\end{array}$ & $\begin{array}{l}\chi^{2}=24,549 \\
P<0,05 \\
\chi^{2}=15,110 \\
P<0,05 \\
\chi^{2}=9,613 \\
P>0,05 \\
\chi^{2}=24,661 \\
P<0,05 \\
\chi^{2}=6,558 \\
P>0,05 \\
\chi^{2}=24,814 \\
P<0,05 \\
\chi^{2}=12,064 \\
P>0,05 \\
\chi^{2}=26,018 \\
P<0,05 \\
\chi^{2}=22,225 \\
P<0,05 \\
\chi^{2}=9,111 \\
P>0,05 \\
\chi^{2}=23,862 \\
P<0,05 \\
\chi^{2}=13,754 \\
P>0,05 \\
\chi^{2}=26,594 \\
P<0,05 \\
\chi^{2}=4,665 \\
P>0,05\end{array}$ & $\begin{array}{l}\chi^{2}=10,671 \\
P>0,05 \\
\chi^{2}=12,700 \\
P>0,05 \\
\chi^{2}=21,757 \\
P<0,05 \\
\chi^{2}=28,117 \\
P<0,05 \\
\chi^{2}=24,173 \\
P<0,05 \\
\chi^{2}=13,187 \\
P>0,05 \\
\chi^{2}=24,205 \\
P<0,05 \\
\chi^{2}=13,744 \\
P>0,05 \\
\chi^{2}=11,379 \\
P>0,05 \\
\chi^{2}=9,136 \\
P>0,05 \\
\chi^{2}=8,681 \\
P>0,05 \\
\chi^{2}=24,817 \\
P<0,05 \\
\chi^{2}=11,702 \\
P>0,05 \\
\chi^{2}=8,953 \\
P>0,05\end{array}$ & $\begin{array}{l}\chi^{2}=37,830 \\
\mathrm{P}<0,05 \\
\chi^{2}=7,588 \\
\mathrm{P}>0,05 \\
\chi^{2}=9,715 \\
\mathrm{P}>0,05 \\
\chi^{2}=2,724 \\
\mathrm{P}>0,05 \\
\chi^{2}=5,192 \\
\mathrm{P}>0,05 \\
\chi^{2}=8,887 \\
\mathrm{P}>0,05 \\
\chi^{2}=23,618 \\
\mathrm{P}<0,05 \\
\chi^{2}=23,717 \\
\mathrm{P}<0,05 \\
\chi^{2}=36,783 \\
\mathrm{P}<0,05 \\
\chi^{2}=9,527 \\
\mathrm{P}>0,05 \\
\chi^{2}=10,900 \\
\mathrm{P}>0,05 \\
\chi^{2}=23,256 \\
\mathrm{P}<0,05 \\
\chi^{2}=24,833 \\
\mathrm{P}<0,05 \\
\chi^{2}=4,296 \\
\mathrm{P}>0,05\end{array}$ \\
\hline \multirow{13}{*}{ Empatía } & $\begin{array}{l}\text { Cuando alguien tiene problemas me } \\
\text { preocupa } \\
\text { Cuando le pegan u ofenden a algún } \\
\text { compañero lo defiendes }\end{array}$ & $\begin{array}{l}\chi^{2}=26,194 \\
P<0,05 \\
\chi^{2}=25,674 \\
P<0,05\end{array}$ & $\begin{array}{l}\chi^{2}=11,781 \\
P>0,05 \\
\chi^{2}=27,069 \\
P<0,05\end{array}$ & $\begin{array}{l}\chi^{2}=37,336 \\
P<0,05 \\
\chi^{2}=10,886 \\
P \quad 0,05\end{array}$ \\
\hline & $\begin{array}{l}\text { Cuando alguien se equivoca o hace algo } \\
\text { incorrecto le corrijo intentando no } \\
\text { ofenderlo }\end{array}$ & $\begin{array}{l}\chi^{2}=24,308 \\
P<0,05\end{array}$ & $\begin{array}{l}\chi^{2}=11,035 \\
P>0,05\end{array}$ & $\begin{array}{l}\chi^{2}=10,528 \\
P>0,05\end{array}$ \\
\hline & Ayudo a los que tienen problemas & $\begin{array}{l}\chi^{2}=11,448 \\
P>0,05\end{array}$ & $\begin{array}{l}\chi^{2}=13,667 \\
P>0,05\end{array}$ & $\begin{array}{l}\chi^{2}=4,100 \\
P>0,05\end{array}$ \\
\hline & $\begin{array}{l}\text { Cuando lo necesitan, animo a mis amigos } \\
\text { y compañeros }\end{array}$ & $\begin{array}{l}\chi^{2}=11,907 \\
P>0,05\end{array}$ & $\begin{array}{l}\chi^{2}=24,430 \\
P<0,05\end{array}$ & $\begin{array}{l}\chi^{2}=40,297 \\
P<0,05\end{array}$ \\
\hline & $\begin{array}{l}\text { Colaboro para que se trabaje mejor y con } \\
\text { más interés }\end{array}$ & $\begin{array}{l}\chi^{2}=10,675 \\
P>0,05\end{array}$ & $\begin{array}{l}\chi^{2}=10,948 \\
P>0,05\end{array}$ & $\begin{array}{l}\chi^{2}=35,625 \\
P<0,05\end{array}$ \\
\hline & $\begin{array}{l}\text { Hablo muy bien, tengo facilidad para } \\
\text { hablar }\end{array}$ & $\begin{array}{l}\chi^{2}=20,862 \\
P<0,05\end{array}$ & $\begin{array}{l}\chi^{2}=13,068 \\
P>0,05\end{array}$ & $\begin{array}{l}\chi^{2}=12,311 \\
P>0,05\end{array}$ \\
\hline & $\begin{array}{l}\text { Me gusta hablar con mis amigos y } \\
\text { compañero }\end{array}$ & $\begin{array}{l}\chi^{2}=10,522 \\
P>0,05\end{array}$ & $\begin{array}{l}\chi^{2}=29,693 \\
P<0,05\end{array}$ & $\begin{array}{l}\chi^{2}=41,458 \\
P<0,05\end{array}$ \\
\hline & Cuando ofendo o molesto, pido disculpas & $\begin{array}{l}\chi^{2}=27,054 \\
P<0,05\end{array}$ & $\begin{array}{l}\chi^{2}=24,363 \\
P<0,05\end{array}$ & $\begin{array}{l}\chi^{2}=23,341 \\
P<0,05\end{array}$ \\
\hline & $\begin{array}{l}\text { Tengo paciencia con los errores o } \\
\text { equivocaciones de los demás }\end{array}$ & $\begin{array}{l}\chi^{2}=12,183 \\
P>0,05\end{array}$ & $\begin{array}{l}\chi^{2}=28,289 \\
P<0,05\end{array}$ & $\begin{array}{l}\chi^{2}=5,951 \\
P>0,05\end{array}$ \\
\hline & $\begin{array}{l}\text { Me intereso por lo que pueda sucederle a } \\
\text { mis compañeros }\end{array}$ & $\begin{array}{l}\chi^{2}=21,077 \\
P<0,05\end{array}$ & $\begin{array}{l}\chi^{2}=13,928 \\
P>0,05\end{array}$ & $\begin{array}{l}\chi^{2}=35,874 \\
P<0,05\end{array}$ \\
\hline & $\begin{array}{l}\text { Recojo lo que los demás tiran o dejan } \\
\text { fuera de su sitio }\end{array}$ & $\begin{array}{l}\chi^{2}=26,244 \\
P<0,05\end{array}$ & $\begin{array}{l}\chi^{2}=5,800 \\
P>0,05\end{array}$ & $\begin{array}{l}\chi^{2}=37,735 \\
P<0,05\end{array}$ \\
\hline & $\begin{array}{l}\text { Cuando alguien es rechazado o ignorado } \\
\text { me acerco a él e intento ayudarlo }\end{array}$ & $\begin{array}{l}\chi^{2}=26,537 \\
P<0,05\end{array}$ & $\begin{array}{l}\chi^{2}=7,280 \\
P>0,05\end{array}$ & $\begin{array}{l}\chi^{2}=5,581 \\
P>0,05\end{array}$ \\
\hline & $\begin{array}{l}\text { Sé cuándo tengo que hablar y cuando } \\
\text { tengo que callar }\end{array}$ & $\begin{array}{l}\chi^{2}=11,600 \\
P>0,05\end{array}$ & $\begin{array}{l}\chi^{2}=13,442 \\
P>0,05\end{array}$ & $\begin{array}{l}\chi^{2}=12,607 \\
P>0,05\end{array}$ \\
\hline
\end{tabular}

Fuente: Contreras, Benítez \& Amaya De Armas (2016).

\section{Autoestima.}

En la habilidad social Autoestima, sólo el constructo "ser capaz de hacer las cosas tan bien como la mayoría de la gente", presentó altos niveles de homogeneidad en las respuestas asociadas a los niveles de desempeños de 


\section{Assensus}

quienes las dieron, en cada una de las tres áreas analizadas (ver $\chi 2$ y valores $P$ $<0,05$ en la tabla 6). El constructo "Estar convencido de tener algunas buenas cualidades" presentó altos niveles de homogeneidad con los niveles de desempeños de quienes las dieron, en dos de las tres áreas analizadas $(\chi 2$ $=27,377$ y $\chi 2=35,899$ para Matemáticas y Ciencias Naturales respectivamente con $\mathrm{P}<0,05)$. Mientras que "sentir que no se tiene demasiadas cosas de las que estar orgulloso" presentó asociaciones estadísticamente significativas sólo con los desempeños en el área de matemáticas. Además, el estado de la habilidad autoestima tiene una tendencia entre en desarrollo a bien desarrollada en los estudiantes de las dos instituciones de la muestra, como puede apreciarse en la información mostrada en la tabla 7.

Tabla 7. Resumen de niveles de desempeño en las tres áreas contra grado de desarrollo del constructo "ser capaz de hacer las cosas tan bien como la mayoría de la gente.

\begin{tabular}{llrrrrrr}
\hline & \multicolumn{6}{c}{$\begin{array}{c}\text { Ser capaz de hacer las cosas tan bien como la } \\
\text { mayoría de la gente }\end{array}$} \\
\cline { 3 - 7 } & & Nunca & Casi Nunca & A veces & Casi siempre & Siempre & Total \\
\hline Notas de & Bajo & 3 & 2 & 8 & 12 & 12 & 37 \\
Matemáticas & Básico & 4 & 2 & 15 & 27 & 35 & 83 \\
& Alto & 0 & 0 & 2 & 9 & 23 & 34 \\
& Superior & 0 & 0 & 0 & 1 & 0 & 1 \\
Total & & 7 & 4 & 25 & 49 & 70 & 155 \\
Notas de & Bajo & 3 & 0 & 12 & 14 & 13 & 42 \\
Lengua & Básico & 3 & 4 & 13 & 27 & 41 & 88 \\
Castellana & Alto & 1 & 0 & 0 & 8 & 14 & 23 \\
& Superior & 0 & 0 & 0 & 0 & 2 & 2 \\
Total & & 7 & 4 & 25 & 49 & 70 & 155 \\
Notas de & Bajo & 2 & 0 & 3 & 4 & 3 & 12 \\
Ciencias & Básico & 4 & 4 & 17 & 26 & 33 & 84 \\
Naturales & Alto & 1 & 0 & 5 & 19 & 34 & 59 \\
\hline Total & & 7 & 4 & 25 & 49 & 70 & 155 \\
\hline
\end{tabular}

Fuente: Contreras, Benítez \& Amaya De Armas (2016).

Los resultados académicos de los estudiantes de ambos grupos muestran una relación positiva con los encontrados para la autoestima, lo que refuerza lo reportado por Mejía, Pastrana y Mejía (2011), en cuanto a que un buen desarrollo de la autoestima favorece el rendimiento académico, y los resultados académicos en las tres áreas evaluadas están alrededor del básico en los de la Institución Educativa San Vicente de Paul y entre básico y alto para los del Centro Educativo Panamericano. Según estos autores, una autoestima bien desarrollada ayuda a los estudiantes a construir un marco de referencia desde el cual puedan interpretar la realidad externa a través de sus experiencias y valores personales, mejorando su rendimiento académico, refuerza sus hábitos saludables, y de esta forma fortalece el desarrollo de su autonomía personal y profesional, por lo que podría esperarse que el desempeño académico de estos estudiantes tienda a mejorar en el resto del bachillerato.

\section{Asertividad.}

En la habilidad social Asertividad, los constructos "cuando se necesita algo se pide el favor", "aceptar que hay muchas cosas que no sabes", "ser capaz de conversar con personas desconocidas" y "cuando tienes dudas, pides que te las 


\section{Assensus}

aclaren" presentó altos niveles de homogeneidad en las respuestas asociadas a los niveles de desempeños de quienes las dieron, sólo en dos de las tres áreas analizadas (ver $\chi 2$ y valores $p<0,05$ en la tabla 6 ). Mientras que los constructos "decir que no cuando le piden algo prestado", "disculparse cuando se comete un error", "aceptar cuando está asustado y se pide ayuda", "si algo te molesta, lo haces saber", "hacer preguntas de tipo personal a los demás", "hacer críticas constructivas a los demás", "poder ser amigo de alguien que te ha rechazado anteriormente" y "ser capaz de exigir ser atendido cuando te han hecho esperar mucho tiempo" presentaron altos niveles de homogeneidad con los desempeños de los estudiantes en sólo una de las tres área que se evaluaron. Además, el estado de la habilidad asertividad es bastante heterogénea en los estudiantes de las dos instituciones de la muestra. Se muestra como ejemplo la información correspondiente al constructo "aceptar que hay muchas cosas que no sabes" mostrada en la tabla 8.

Tabla 8. Resumen de niveles de desempeño en las tres áreas contra grado de desarrollo del constructo "aceptar que hay muchas cosas que no sabes".

\begin{tabular}{llrrrrrr}
\hline & \multicolumn{7}{c}{ Aceptar que hay muchas cosas que no sabes } \\
& & Nunca & Casi Nunca & A veces & Casi siempre & Siempre & Total \\
\hline Notas de & Bajo & 5 & 1 & 9 & 7 & 15 & 37 \\
matemáticas & Básico & 4 & 7 & 17 & 28 & 27 & 83 \\
& Alto & 2 & 7 & 4 & 6 & 15 & 34 \\
& Superior & 0 & 0 & 1 & 0 & 0 & 1 \\
Total & & 11 & 31 & 41 & 57 & 155 \\
Notas de & Bajo & 4 & 5 & 9 & 8 & 16 & 42 \\
castellano & Básico & 6 & 5 & 20 & 27 & 30 & 88 \\
& Alto & 1 & 5 & 2 & 6 & 9 & 23 \\
Total & Superior & 0 & 0 & 0 & 0 & 2 & 2 \\
Notas de & & 11 & 15 & 31 & 41 & 57 & 155 \\
ciencias & Bajo & 1 & 1 & 0 & 4 & 6 & 12 \\
naturales & Básico & 8 & 6 & 20 & 22 & 28 & 84 \\
\hline Total & Alto & 2 & 8 & 11 & 15 & 23 & 59 \\
\hline
\end{tabular}

Fuente: Contreras, Benítez \& Amaya De Armas (2016).

De la información recabada también puede inferirse que los resultados académicos de los estudiantes de ambos grupos en las tres áreas evaluadas están alrededor del básico, pero no muestran una relación significativa con la asertividad, ya que el estado de desarrollo de esta habilidad muestra un alto grado de heteroneneidad, tanto intra, como inter grupos, lo que puede interpretarse como que el conocimiento de los derechos propios y la opción de defenderlos, y el reconocimiento y respeto de los derechos de los demás, no tiene una incidencia directa en el rendimiento académico de los estudiantes de las instituciones de la muestra (Monjas et al., 1998).

\section{Empatía.}

En la habilidad social Empatía, sólo el constructo "cuando ofendo o molesto a alguien, pido disculpas", presentó altos niveles de homogeneidad en las respuestas asociadas a los niveles de desempeños de quienes las dieron, en cada una de las tres áreas analizadas (ver $\chi 2$ y valores $P<0,05$ en la tabla 6 ). Entre los constructos "cuando alguien tiene problemas me preocupa", "cuando le 


\section{Assensus}

pegan u ofenden a algún compañero lo defiendes", "cuando lo necesitan, animo a mis amigos y compañeros", "me gusta hablar con mis amigos y compañero", "me intereso por lo que pueda sucederle a mis compañeros" se encontró relación estadísticamente significativa con los niveles de desempeños de los estudiantes sólo en dos de las tres áreas analizadas (ver $\chi 2$ y valores $p<0,05$ en la tabla 6 ). Mientras que los constructos "cuando alguien se equivoca o hace algo incorrecto le corrijo intentando no ofenderlo", "hablo muy bien, tengo facilidad para hablar", "tengo paciencia con los errores o equivocaciones de los demás", "recojo lo que los demás tiran o dejan fuera de su sitio" y "cuando alguien es rechazado o ignorado me acerco a él e intento ayudarlo" presentaron altos niveles de homogeneidad sólo con los desempeños de los estudiante en una de las tres áreas evaluadas como puede apreciarse en la tabla 6 . Además, el estado de la habilidad Empatía tiene una tendencia entre en desarrollo a bien desarrollada en los estudiantes de las dos instituciones de la muestra, como puede apreciarse en la información mostrada en la tabla 9.

Tabla 9. Resumen de niveles de desempeño en las tres áreas contra grado de desarrollo del constructo "cuando ofendo o molesto, pido disculpas".

\begin{tabular}{llrrrrrr}
\hline & \multicolumn{6}{c}{ Cuando ofendo o molesto, pido disculpas } \\
& & Nunca & Casi Nunca & A veces & Casi siempre & Siempre & Total \\
\hline Notas de & Bajo & 2 & 1 & 11 & 6 & 17 & 37 \\
matemáticas & Básico & 2 & 6 & 12 & 14 & 49 & 83 \\
& Alto & 2 & 1 & 4 & 12 & 15 & 34 \\
& Superior & 0 & 0 & 1 & 0 & 0 & 1 \\
Total & & 6 & 8 & 28 & 32 & 81 & 155 \\
Notas de & Bajo & 1 & 5 & 9 & 5 & 22 & 42 \\
castellano & Básico & 5 & 1 & 14 & 23 & 45 & 88 \\
& Alto & 0 & 2 & 5 & 4 & 12 & 23 \\
Total & Superior & 0 & 0 & 0 & 0 & 2 & 2 \\
Notas de & Bajo & 6 & 8 & 28 & 32 & 81 & 155 \\
ciencias & Básico & 2 & 1 & 3 & 1 & 5 & 12 \\
naturales & Alto & 2 & 5 & 19 & 14 & 44 & 84 \\
\hline Total & & 6 & 2 & 6 & 17 & 32 & 59 \\
\hline
\end{tabular}

Fuente: Contreras, Benítez \& Amaya De Armas (2016).

Los resultados académicos evidencian que los desempeños de los estudiantes en las tres asignaturas evaluadas están alrededor de básico, mientras el estado de la habilidad empatía tiene una clara tendencia entre en desarrollo y desarrollado, lo que deja ver cierta coherencia entre los procesos afectivos de la persona al comprender los sentimientos que afectan a otras personas (Rey, 2003) con los desempeños académicos del grupo, por lo que, a futuro, pudieran esperarse mejoras académicas en este grupo de estudiantes.

\section{Conclusiones}

Los resultados evidencian que el nivel de desarrollo de las habilidades sociales: empatía, asertividad y trabajo en equipo de los estudiantes de grado sexto de las Instituciones Educativas de la muestra es bastante heterogéneo entre habilidades: trabajo en equipo y asertividad son bastante heterogéneas tanto intra 


\section{Assensus}

como inter grupos, es decir, su nivel de desarrollo no guarda una tendencia clara en ninguno de los dos grupos, mientras que autoestima y empatía tienen una tendencia entre en desarrollo a bien desarrollada.

Los resultados académicos evidencian desempeños de los estudiantes en las tres asignaturas alrededor de básico para los de San Vicente de Paúl y entre básico y alto para los del Centro Educativo Liceo Panamericano, lo que guarda cierta relación con el estado de habilidades como Autoestima y Empatía, las cuales tienen una clara tendencia entre en desarrollo y desarrolladas, mientras no muestran una clara asociación con habilidades como Asertividad y Trabajo en equipo ya que el estado de desarrollo de estas habilidades muestran un alto grado de heteroneneidad, tanto intra, como inter grupos, es decir, no hay una tendencia marcada al interior de los grupos para ninguna de estas dos habilidades.

Se encontró asociación estadísticamente significativa entre los desempeños logrados por los estudiantes en las áreas de matemáticas y de Ciencias Naturales, con la institución de donde provenían los estudiantes, lo que quiere decir que las calificaciones de los estudiantes de cada institución tendieron a agruparse alrededor de los mismos niveles de desempeño, pero diferenciables entre los grupo, ligeramente mejores para los del Centro Educativo Liceo Panamericano.

\section{Referencias Bibliográficas}

Balseiro, C., Zárate, R., Matus, R., Balan, C., Sacristán, F., García, M \& Pérez, A. (2012). Inserción laboral, desarrollo profesional y desempeño institucional de las (os) egresadas (os) del Plan Único de Especialización en Enfermería de la ENEO-UNAM: una experiencia de doce años. Revista Enfermería universitaria, 9(1), 16-26.

Creswell, J. (2009). Research design: qualitative, quantitative and mixed methods approaches. Thousand Oaks, CA: Sage.

Damián, M. \& Muria, I. (2006). La educación primacía de la familia mediatizada por las instituciones educativas. Psicologia para América Latina, (6) Recuperado el 05 de junio de 2016, de http://pepsic.bvsalud.org/scielo.php?script=sci_arttext\&pid=S1870350X2006000200010\&lng=pt\&tIng=es

Durán, D., Flores, M. \& Valdebenito, V. (2015). Tutoría entre iguales. Concepto y práctica como metodología para la educación inclusiva. Revista de Latinoamericana de Educación inclusiva, 9(2), 23-40.

García, F., Castellón, J., Jiménez, I., Muñoz, M., Monjas, M., Sureda, I., Ferrá, P., Martín, L., Marande, G \& SAnchiz, M. (2013). Aulas como contextos de aceptación y apoyo para integrar a los alumnos rechazados. Apuntes de Psicología Colegio Oficial de Psicología de Andalucía Occidental, 31(2), 145-154.

García, J. González, M. y Ballesteros V. (2001). Introducción a la investigación en educación (Tomo I). Madrid: UNED.

Gil-Pascual, J. (2009). Metodología cuantitativa en educación. Madrid: editorial Uned. 


\section{Assensus}

Godino, J. (2013). Indicadores de idoneidad didáctica de procesos de enseñanza y aprendizaje de las matemáticas. Cuadernos de Investigación y Formación en Educación Matemática, 8 (11), 111132.

Hernández, R., Fernández, C. \& Baptista, L. (2010). Metodología de la investigación (5ta ed.). México, D.F.: McGraw-Hill.

López, A. (2009). Modelo de evaluación continúa formativa-formadora-reguladora y tutorización continua con soporte multimedia apoyado en una plataforma virtual. Tesis doctoral, Madrid: Uned.

Mejía, A., Pastrana, J. \& Mejía, J. (2011). La autoestima, factor fundamental para el desarrollo de la autonomía personal y profesional. XII Congreso internacional de teoría de la educación (Barcelona).

Monjas, M. (2000). Programa de enseñanza de habilidades de interacción social (PEHIS) para niños y niñas en edad escolar. Madrid: CEPE.

Monjas, M. (2014). Aplicaciones de la psicología positiva a la convivencia: bienestar, crecimiento personal y emocional. Recuperado el 14 de junio de 2016, del sitio web: http://recursos.crfptic.es:9080/jspui/bitstream/recursos/558/4/Relaciones_interpersonales_p ositivas.pdf.

Monjas, M., De Benito, M., Casado, M., Conde, M., Peinado, L. \& Rodríguez, M. (1998). Las habilidades sociales en el currículo. Madrid: Ministerio de Educación Cultura y Deportes.

Monjas, M. \& González, B. (2000). Las habilidades sociales en el currículo. Madrid: Ministerio de Educación Cultura y Deportes.

Ortega, J. (2005). Pedagogía social y pedagogía escolar: la educación social en la escuela. Revista de Educación (336), 111-127.

Pascual, A. (2000). Violencia, paz y conflicto en el discurso y la praxis pedagógica. Revista de Pedagogía, 34, 47-82.

Rey, C. (2003). La medición de la empatía en preadolescentes y adolescentes varones: adaptación y validación de una escala. Revista Latinoamericana de Psicología, 35(2), 185-194.

Trujillo, F. (2011). Enfoque de competencias en la educación: del conocimiento al uso ya apropiación. Recuperado el 10 de mayo de 2016, de http://www.colombiaaprende.edu.co/html/familia/1597/article-73369.html

Unesco. (2008). Hacia las sociedades del conocimiento. Informe mundial de la UNESCO. Ediciones UNESCO. Consultado el 10 de mayo de 2016 del sitio web: http://unesdoc.unesco.org/images/0014/001419/141908s.pdf 